\title{
The role of biomechanical factors in ankylosing spondylitis: the patient's perspective
}

\author{
R.C. Ansell ${ }^{1,4}$, T. Shuto ${ }^{2}$, N. Busquets-Perez ${ }^{3}$, E.M.A. Hensor ${ }^{1,4}$, \\ H. Marzo-Ortega ${ }^{1,4}$, D. McGonagle ${ }^{1,4}$ \\ 'Leeds Institute of Rheumatic and Musculoskeletal Medicine, University of Leeds, UK; \\ 2Department of Rheumatology and Orthopaedics, Chiyoda Hospital, Miyazaki, Japan; \\ ${ }^{3}$ Department of Rheumatology, Hospital de Granollers, Barcelona, Spain; ${ }^{4}$ National Institute for Health \\ Research, Leeds Musculoskeletal Biomedical Research Unit, Leeds, UK
}

\begin{abstract}
SUMMARY
Biomechanical factors including occupational joint physical stressing and joint injury have been linked to spondyloarthritis. We explored such factors in ankylosing spondylitis (AS).

A retrospective, online survey was developed alongside the UK National Ankylosing Spondylitis Society (NASS). Questions on early entheseal symptoms, potential precipitating trauma, sporting activity, and physiotherapy were asked.

A total of 1026 patients responded with $44 \%$ recalling an instance of injury or trauma as a potential trigger for their AS. After symptom onset, 55\% modified sporting activities and 28\% reported that the initial AS recommended exercises exacerbated symptoms.

Patients report physical trauma, exercise and physiotherapy as potential triggers for AS symptoms. These findings further support the experimental evidence for the role of biomechanical factors in disease.
\end{abstract}

Key words: Ankylosing spondylitis; enthesitis; injury; exercise; mechanics; physiotherapy; exercise.

Reumatismo, 2015; 67 (3): 91-96

\section{INTRODUCTION}

A nkylosing spondylitis (AS) is the prototypical spondyloarthropathy (SpA), characteristically presenting with low back pain and stiffness. AS is associated with enthesitis/osteitis that can afflict any insertional location, though it is particularly common in the spinal entheses (1). The prevalence and factors associated with the development of enthesitis are poorly understood in early AS. However, as early as the 1950s, a biomechanical theory of enthesitis as a result of trauma to insertional locations had been proposed (2).

There is considerable evidence linking joint injury with the development of psoriatic arthritis (PsA) (3-6) - another disease entity within the SpA family. An association between mechanical factors including recalled joint injury and occupations that involve heavy lifting has been epidemiologically linked to PsA (7). Work in animal models has demonstrated that biomechanical perturbations alone can be associated with the development of an enthesealbased SpA-like immunopathology (8-10). We hypothesised that increased joint mechanical stressing, physical injury or trauma could cause a biomechanical disturbance with the subsequent development of SpA or AS.

A further possibility is that exercise - either independently achieved, or recommended by medical practitioners - might lead to increased tissue biomechanical stressing. The potential role of injury or skeletal biomechanical related factors, especially exercise, as an early entheseal-related pathology initiator in AS remains poorly understood. A rigorous physiotherapy regime has formed the cornerstone of therapy for $\overline{\text { Corresponding author: }}$ Dennis McGonagle

Leeds Institute of Rheumatic and Musculoskeletal Medicine

University of Leeds, Chapel Allerton Hospital, Chapeltown Road, Leeds, LS7 4SA, UK. E-mail: D.G.McGonagle@leeds.ac.uk 
AS for several decades (11). This has been shown to be of clear benefit to many patients and is recommended by national and international guidelines $(12,13)$.

To our knowledge, no studies have addressed the issue of whether or not exercise could in some patients be associated with the paradoxical exacerbation of disease. This is highly relevant to patients who have to decide whether to pursue the physiotherapy regime suggested by their medical practitioners.

We explored issues relating to the prevalence of enthesitis in the early phases of $\mathrm{AS}$ - predating the onset of low back pain - and its possible relationship with injury and physical activity levels.

\section{MATERIALS AND METHODS}

The National Ankylosing Spondylitis Society (NASS) is a UK charity representing patients with ankylosing spondylitis and allied conditions, active in the promotion of research into these conditions. Following an agenda set by patient research partners who are members of NASS, questions were devised by rheumatologists and subsequently reviewed by NASS board members including patient representatives. As the survey was to be completed by patients rather than clinicians, wherever possible layman's language was used rather than medical terminology (for example, knee cap rather than patella).

An online questionnaire was then created (www.surveymonkey.com, Palo Alto, California, USA) and disseminated by NASS to their members via email. Questions concerned:

1) gender; age at diagnosis;

2) HLA-B27 status (if known); history of skin psoriasis and other SpA associated diseases (uveitis, inflammatory bowel disease);

3) patient recall of entheseal features prior to diagnosis - heel pain, knee cap pain, chest wall pain;

4) patient recall of perceived trauma - incidence of trauma, timing of trauma in relation to onset of inflammatory back pain;
5) patient experience of exercise participation after diagnosis;

6) patient experience of physiotherapy and recommended exercises (Supplementary file).

All answers received related to the recall and reports of the patients themselves rather than those of their medical practitioner.

Responses were received electronically and anonymously; consequently, queries raised by the responses of individual participants could not be addressed. Given that this study focuses on patient perception, responses with missing data have been included, since patients may not always be aware of factors such as HLA-B27 status. Statistical analysis was performed using SPSS version 21. Basic descriptive analysis was applied to all variables. In addition, Chi-squared testing was performed to ascertain differences between groups with and without psoriasis, and HLA-B27 positive and negative groups.

Data were owned and stored securely by NASS and anonymised data was released to the investigators for analysis.

\section{RESULTS}

A total of 1026 responses were received from a total NASS readership of 3000 potential respondents over the summer of 2012 [63.3\% male $(n=653)]$. Of these, $59.5 \%$ of respondents knew they were HLA-B27 positive; $8.8 \%$ knew they were HLA-B27 negative, with the remainder unsure. The peak age bracket for diagnosis was $26-30$ years. $26.4 \%$ of respondents reported a definite past or present history of psoriasis.

\section{Enthesitis}

Table I illustrates patient recollection of symptoms of enthesitis. For each manifestation, the majority of respondents recalled entheseal symptoms starting more than two years before the onset of back pain. Pearson chi-squared analysis showed no significant difference between recollection of entheseal symptoms in HLA-B27 positive and negative respondents. There was a 
Table I - Possible early symptoms of entheseal disease reported by participants.

\begin{tabular}{|l|c|c|c|}
\hline & $\begin{array}{c}\text { Yes } \\
\%(\mathbf{n})\end{array}$ & $\begin{array}{c}\text { No } \\
\%(\mathbf{n})\end{array}$ & $\begin{array}{c}\text { Not sure/Don't } \\
\text { know \% (n) }\end{array}$ \\
\hline $\begin{array}{l}\text { Ever had heel pain prior to onset of } \\
\text { back pain ( } \mathrm{n}=1026)\end{array}$ & $27.5 \%(282)$ & $54.1 \%(555)$ & $18.4 \%(189)$ \\
\hline $\begin{array}{l}\text { Ever had knee cap pain prior to } \\
\text { onset of back pain ( } \mathrm{n}=1022)\end{array}$ & $30.9 \%(316)$ & $56.4 \%(576)$ & $12.7 \%(130)$ \\
\hline $\begin{array}{l}\text { Ever had pain and stiffness around } \\
\text { the chest/chest wall prior to onset } \\
\text { of back pain }(\mathrm{n}=1022)\end{array}$ & $25.2 \%(258)$ & $61.1 \%(624)$ & $13.7 \%(140)$ \\
\hline
\end{tabular}

Table II - Patient-estimated temporal lag between occurrence of trauma and onset of symptoms of ankylosing spondylitis in those patients reporting an incidence of trauma prior to symptoms onset (total $n=452$ ).

\begin{tabular}{|l|c|}
\hline Estimated time frame & $\%(\mathbf{n})$ \\
\hline Days before & $13.3 \%(60)$ \\
\hline Weeks before & $16.4 \%(74)$ \\
\hline Months before & $28.3 \%(128)$ \\
\hline Years before & $40.5 \%(183)$ \\
\hline Not answered & $1.5 \%(7)$ \\
\hline
\end{tabular}

significant difference between recollection of heel pain prior to diagnosis with AS in patients with and without skin psoriasis $(82 / 211$ and $174 / 583$ respectively; $p=0.04)$. This difference was not observed for any of the other entheseal symptoms recorded in groups with and without psoriasis.

\section{Recall of trauma}

Overall, $44.1 \%(n=452)$ recalled a prior incident of trauma that they felt triggered their disease. $40.7 \%$ could not recall any incidence of trauma, with the remaining fraction either unsure $(14.3 \%, n=147)$ or skipping the questions $(<1 \%)$. The temporal relationship with disease onset is shown in Table II; over $50 \%$ of those who recalled trauma felt that it had occurred in the days, weeks or months before their diagnosis with AS.

\section{Sporting activity}

The majority of respondents [61.4\% $(\mathrm{n}=630)]$ reported being quite active or very active in sport prior to diagnosis of AS. Among these respondents, sporting habits changed following the diagnosis of AS in multiple ways (Figure 1). A total of 569 respondents reported that they had made adjustments to their participation in sport following diagnosis of AS. Some respondents had reduced their sporting activity in more than one way; in total, this group of patients made 764 modifications to their sporting activity.

\section{Perception of the effect of physiotherapy}

A quarter of the respondents [27.7\% $(n=284)$ ] felt that the original exercises recommended for AS had led to a flare in their symptoms. 32.4\% (332/1026) of respondents reported that they had felt it necessary to change the recommended exercises for their AS to lessen disease flares. The majority [58.2\% (597/1026)] had not had to modify their exercises; the remaining respondents either skipped the question or were unsure.

\section{DISCUSSION}

The survey findings showed that symptoms that may be compatible with peripheral enthesitis are not uncommon in patients who subsequently develop AS, and these symptoms may precede the onset of axial or spinal pain by several years. Joint injury (in the form of an incidence of trauma), exercise and/or physiotherapy were reportedly associated with either the onset of disease 


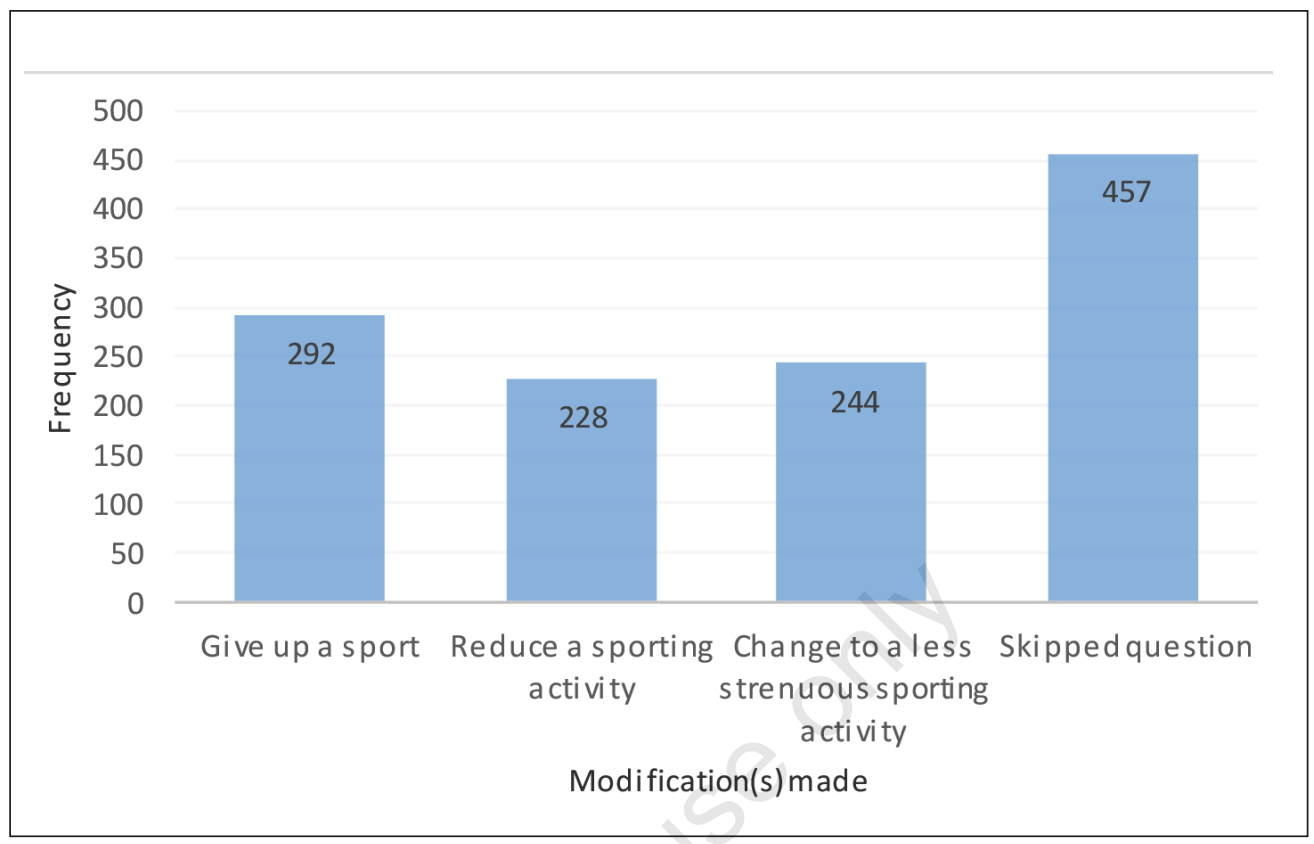

Figure 1 - Reported changes to sporting activity following diagnosis with ankylosing spondylitis. Respondents were asked to select all options that applied, or to skip the question. Some respondents selected more than one option, hence total reported number of modifications $=764$.

or exacerbating disease in a substantive proportion of respondents. Many respondents reported a change in sporting activity following the diagnosis of AS. This has important implications for participation in healthy lifestyles. Biomechanical perturbation has been experimentally shown to lead to the development of an SpA-type enthesitis. The findings of perception of disease triggering by an incidence of physical trauma are in keeping with this.

The finding that some patients experience a worsening in symptoms after physiotherapy may also reflect a role for ongoing biomechanical perturbation in the perpetuation of active disease.

Decreased sporting participation may further imply this, although this latter finding is more difficult, as other reasons for reduced independent exercise (i.e., physical activity outwith that of conventional physiotherapy) were not addressed by the study. Nonetheless, this survey suggests that biomechanical perturbation can take many forms, with the same outcome of worsened pain and active inflammatory disease.
The retrospective, patient-reported survey has several limitations. Firstly, although NASS is the UK's leading charity for AS and axial $\mathrm{SpA}$, it is possible that some members do not have a formal diagnosis of AS/axial SpA but still completed the questionnaire. However, our survey did ask for disease duration and at what age a diagnosis was received. These measures go some way towards minimising the chance of people outside of the target population participating in the survey. We feel that any participants without a formal diagnosis will be few in number.

Secondly, this retrospective study may be prone to recall bias.

It will be necessary to ascertain these findings in longitudinal cohorts of patients presenting with enthesitis. However, these findings suggest that questions about previous possible entheseal symptoms may be of use in diagnosing axial SpA.

The present ASAS classification criteria for SpA include the presence of enthesitis at the heel (14), but not enthesitis at other sites. 
A final limitation of this study is the absence of a control group. This work was conceived in partnership with NASS and is a springboard to further research. It would be interesting to repeat this work among patients with diseases not supported by the enthesitis hypothesis, for example seropositive rheumatoid arthritis.

The recollections of the respondents raise the possibility that previous injury may be a factor in the initiation of AS. Certainly, there was a significant patient perception of trauma as the precipitant for disease. The notion of trauma was deliberately wideranging and not specific, reflecting the concept that an apparently minor injury might have significant implications later on, most notably in psoriatic arthritis (2-5). That vigorous exercise might exacerbate symptoms of AS with at least a subset of patients is supported by observations in the field of psoriatic arthritis (4-6). However, the temporal lag between perceived trauma and symptom onset was wide-ranging; most respondents recalling trauma reported that it had occurred months or years before the symptom onset.

Mechanistically it is easier to envisage a link between recent trauma and AS onset; future studies should address this. Magnetic resonance imaging has reported peri-entheseal bone lesions akin to $\mathrm{SpA}$ in normal subjects and following exercise (12-15). Whether it is mechanical stressing per se, injury related to stressing including micro-injury or a combination of both has not been determined.

\section{CONCLUSIONS}

Perhaps the most contentious result from this survey is the findings surrounding physiotherapy and AS, where $27.7 \%$ of respondents reported exercise-related disease flares and $32.4 \%$ had felt it necessary to modify their recommended exercises to lessen disease flares. Interestingly, the number of patients modifying their exercises outnumbered those reporting symptom flare following exercise. The survey did not address potential reasons for this unexpected finding.
Our findings point to the important role of enthesitis in early AS in some patients. The findings also raise issues relevant to the role of joint injury and biomechanics in the AS disease process. However, the survey is limited by recall bias.

With exercise a cornerstone of treatment of AS, these findings highlight the need for appropriate exercises in the right setting. These results should thus be confirmed in longitudinal studies and evaluated in early inception cohorts.

Acknowledgements: we are extremely grateful to the members of the UK National Ankylosing Spondylitis Society (NASS) for their responses to the survey, and to the staff and board members of NASS for their assistance in the process of this work.

\section{REFERENCES}

1. McGonagle D, Emery P. Enthesitis, osteitis, microbes, biomechanics, and immune reactivity in ankylosing spondylitis. J Rheumatol. 2000; 27: 2302-4.

2. La Cava G. Enthesitis; traumatic disease of insertions. JAMA. 1959; 169: 254-5.

3. Olivieri I, Padula A, D’Angelo S, Scarpa R. Role of trauma in psoriatic arthritis. J Rheumatol. 2008; 35: 2085-7.

4. Langevitz P, Buskila D, Gladman DD. Psoriatic arthritis precipitated by physical trauma. J Rheumatol. 1990; 17: 695-7.

5. Goupille P, Soutif D, Valat JP. Psoriatic arthritis precipitated by physical trauma. J Rheumatol. 1991 ; $18: 633$.

6. Pattison E, Harrison BJ, Griffiths CE, Silman AJ, Bruce IN. Environmental risk factors for the development of psoriatic arthritis: results from a case-control study. Ann Rheum Dis. 2008; 67: 672-6.

7. Eder L, Law T, Chandran V, Shanmugarajah S, Shen H, Rosen CF, et al. Association between environmental factors and onset of psoriatic arthritis in patients with psoriasis. Arthritis Care Res (Hoboken). 2011; 63: 1091-7.

8. Jacques P, Lambrecht S, Verheugen E, Lories R, Kollias G, Verbruggen G, et al. Biomechanical stretch is a key initiating event in the development of enthesitis in murine spondyloarthritis. Clin Exp Rheumatol. 2009; 28: 606.

9. McGonagle D, Thomas RC, Schett G. Spondyloarthritis: may the force be with you? Ann Rheum Dis. 2014; 73: 321-3.

10. Jacques P, McGonagle D. The role of mechanical stress in the pathogenesis of spondyloar- 
thritis and how to combat it. Best Pract Res Clin Rheumatol. 2014; 28: 703-10.

11. Kjeken I, Dagfinrud H, Mowinckel P, et al. Rheumatology care: Involvement in medical decisions, received information, satisfaction with care, and unmet health care needs in patients with rheumatoid arthritis and ankylosing spondylitis. Arthritis Rheum. 2006; 55: 394-401.

12. van den Berg R, Baraliakos X, Braun J, van der Heijde D. First update of the current evidence for the management of ankylosing spondylitis with non-pharmacological treatment and nonbiologic drugs: a systematic literature review for the ASAS/EULAR management recom- mendations in ankylosing spondylitis. Rheumatology (Oxford). 2012; 51: 1388-96.

13. Zochling J, van der Heijde D, Dougados M, Braun J. Current evidence for the management of ankylosing spondylitis: a systematic literature review for the ASAS/EULAR management recommendations in ankylosing spondylitis. Ann Rheum Dis. 2006; 65: 42332.

14. Sieper J, Rudwaleit M, Baraliakos X, Brandt J, Braun J, Burgos-Vargas R, et al. The Assessment of SpondyloArthritis international Society (ASAS) handbook: a guide to assess spondyloarthritis. Ann Rheum Dis. 2009; 68 (Suppl. 2): ii1-44. 